\title{
Enhanced Orthogonal Matching Pursuit Algorithm and Its Application in Mechanical Equipment Fault Diagnosis
}

\author{
Yong Lv, Jie Luo, and Cancan Yi \\ The Key Laboratory of Metallurgical Equipment and Control of Education Ministry, Wuhan University of Science and Technology, \\ Wuhan 430081, China \\ Correspondence should be addressed to Jie Luo; luojie19870228@hotmail.com
}

Received 29 May 2017; Accepted 30 July 2017; Published 7 September 2017

Academic Editor: Giosuè Boscato

Copyright (C) 2017 Yong Lv et al. This is an open access article distributed under the Creative Commons Attribution License, which permits unrestricted use, distribution, and reproduction in any medium, provided the original work is properly cited.

\begin{abstract}
The vibration signal measured from the mechanical equipment is associated with the operation of key structure, such as the rolling bearing and gear. The effective signal processing method for early weak fault has attracted much attention and it is of vital importance in mechanical fault monitoring and diagnosis. The recently proposed atomic sparse decomposition algorithm is performed around overcomplete dictionary instead of the traditional signal analysis method using orthogonal basis operator. This algorithm has been proved to be effective in extracting useful components from complex signal by reducing influence of background noises. In this paper, an improved linear frequency-modulated (Ilfm) function as an atom is employed in the proposed enhanced orthogonal matching pursuit (EOMP) algorithm. Then, quantum genetic algorithm (QGA) with the OMP algorithm is integrated since the QGA can quickly obtain the global optimal solution of multiple parameters for rapidly and accurately extracting fault characteristic information from the vibration signal. The proposed method in this paper is superior to the traditional OMP algorithm in terms of accuracy and reducing the computation time through analyzing the simulation data and real world data. The experimental results based on the application of gear and bearing fault diagnosis indicate that it is more effective than traditional method in extracting fault characteristic information.
\end{abstract}

\section{Introduction}

According to the statistics, the fault derived from the mechanical parts, such as the shaft and the gear, mainly causes mechanical accidents. Commonly, these faults are often accompanied by nonlinear vibration phenomenon and its vibration signals are often complicated as the strong background noise [1]. Effective analysis method for vibration signal is the focus of much research in mechanical fault monitoring and diagnosis. In order to realize the mechanical fault monitoring and intelligent diagnosis, the method of feature extraction is widely adopted.

In view of nonstationary and nonlinear signal processing, many studies have been implemented. Gabor [2] proposed the STFT (short-time Fourier transform), which overcomes the inadequacy of the Fourier transform. However, it is unsuitable for dynamic signals due to the fixed time window width. Mallat [3] proposed the WT (wavelet transform), which overcomes the shortcoming that the window does not change with frequency. However, it lacks self-adaptability, because the results are closely related to the choice of wavelet basis function. Then, in order to completely reduce the limitations of time-frequency analysis method and better analyze the local time-frequency characteristics of nonstationary or nonlinear signals, Huang et al. [4] presented EMD (Empirical Mode Decomposition). It owns the advantages of orthogonality and completeness and has been widely applied in the fields of biomedical engineering, mechanical fault diagnosis, and analysis of seismic signal [5-7]. However, it creates problems such as envelope, owe envelope [8], mode mixing [9], and end effects [10].

The most traditional signal analysis methods are based on the inner product operation using orthogonal basis. Thus, it tries to use a fixed base function or the same base function of the properties that represents arbitrary signals and ignores the characteristics of the signal itself. Moreover, the energy of the decomposed signal will be distributed on different bases because of the orthogonality. This characteristic is bad for the signal recognition and compression. In order to achieve the fact that the representation of signal is self-adaptive, 
more flexible, and concise, Mallat and Zhang [11] proposed the sparse decomposition and created a novel method of signal sparse representation in view of wavelet analysis. It breaks the traditional ways of orthogonal basis selection and decomposes the signal into the linear combination of a series of the basic signal (atoms), in which overcomplete dictionary of atoms replaces orthogonal basis. It can not only efficiently realize the representation of signal, but also reflect the intrinsic characteristics of signal. Since this approach has advantages of self-adaptability and flexibility, it is widely used in the fields of image processing $[12,13]$, biomedical engineering $[14,15]$, communication engineering [16], and seismic signal processing $[17,18]$. To date, through many years of development in signal sparse representation, many decomposition algorithms have been proposed, such as the MP (Matching Pursuit) algorithm, the OMP (Orthogonal MP) algorithm, the BP (Basis Pursuit) algorithm [19], the GBP (Greedy BP) algorithm [20], the ROMP (Regularized Orthogonal MP) algorithm [21, 22], the Co-SaMP (Compressive Sampling MP) algorithm [23], and the StOMP (Stage wise Orthogonal MP) algorithm [24].

In view of the sparse theory, scholars have done some researches of fault diagnosis. Candes et al. [25] presented the thought of stage wise matching pursuit and then proposed the line frequency modulation wavelet path tracking method. On the support of linear frequency modulation based wavelet path tracing method, Peng et al. [26] proposed a signal sparse decomposition method based on multiscale line frequency modulation. However, there exists problems like loss of local signal amplitude information, huge computation and decomposition of low efficiency, and so on. Moreover, the researchers have combined sparse theory with the other theories and presented many new methods for fault diagnosis [27, 28]. Wang et al. [29] combined minimum entropy solution of convolution with sparse decomposition for feature extraction of the weak fault. Similarly, Yan and Zhou [30] combined relevant cumulant with StOMP algorithm for feature extraction of the weak impulse signal. The computation efficiency is higher than traditional StOMP algorithm, but the performance of the StOMP algorithm is always dependent on the threshold selection. Taking all the above factors into consideration, the OMP algorithm is used in this paper.

In this paper, the enhanced orthogonal matching pursuit (EOMP) algorithm firstly uses an improved linear frequencymodulated (Ilfm) function as atoms, which can improve the precision and computing efficiency of reconstructing signal. Then it combines quantum genetic algorithm (QGA) with the OMP algorithm, as the QGA can quickly solve multiple parameters of the global optimal solution problem. It can further increase the calculation efficiency of the OMP algorithm. Thus, fault features extraction from vibration signal can be recognized. The EOMP algorithm is superior to traditional OMP algorithms on the accuracy and computation efficiency in the analysis of simulation data and experimental signal. It has been proved that the EOMP algorithms are more feasible and effective in real mechanical equipment fault diagnosis.

\section{Algorithm Description}

2.1. The OMP Algorithm. The MP algorithm belongs to the greedy iterative algorithm. In each iteration, it selects atoms from overcomplete dictionary that best matches with the structure of source signal. Then it requires only a small part of the atoms to accurately represent the source signal until the convergence by multiple iterations. The OMP algorithm is proposed by Pati et al. [31]. Naturally, it is consistent with the MP algorithm on the general idea. However, it adds the atoms orthogonalization to the algorithm, which makes the convergence of the OMP algorithm faster and better. The basic process of the OMP algorithm is briefly introduced as follows.

Given a source signal $x \in R^{N}$, where $N$ is the length of the signal space, $r_{0}=x$. Define a dictionary of atoms $D=$ $\left\{g_{\gamma}\right\}_{\gamma \in \Gamma}$, where $\Gamma$ is a set of $\gamma,\left\|g_{\gamma}\right\|=1$. The source signal is decomposed as follows:

$$
r_{0}=\left\langle g_{\gamma_{0}}, r_{0}\right\rangle g_{\gamma_{0}}+r_{1}
$$

where $\left\langle g_{\gamma_{0}}, r_{0}\right\rangle g_{\gamma_{0}}$ is the projection of $r_{0}$ to $g_{\gamma_{0}}$ and $r_{1}$ is the residual error after the first iteration. Obviously, $g_{\gamma_{0}}$ is obviously orthogonal to $r_{1}$. Hence, there exists the following equation for the decomposition of conservation of energy:

$$
\left\|r_{0}\right\|^{2}=\left|\left\langle g_{\gamma_{0}}, r_{0}\right\rangle\right|^{2}+\left\|r_{1}\right\|^{2}
$$

If the energy of the residual $\left\|r_{1}\right\|^{2}$ is minimized, $\left|\left\langle g_{\gamma_{0}}, r_{0}\right\rangle\right|$ becomes the maximum. This indicates that the best atom is related to the largest inner product of the signal $x$ and the selected atom.

For the MP algorithm, after $M$ iterations, the signal $x$ can be represented as

$$
x=\sum_{m=0}^{M-1}\left\langle g_{\gamma_{m}}, r_{m}\right\rangle g_{\gamma_{m}}+r_{m} .
$$

However, in the OMP algorithm, the selected atom $g_{\gamma_{m}}$ needs to be transformed to Schmidt orthonormalization as follows:

$$
u_{m}=g_{\gamma_{m}}-\sum_{k=0}^{M-1} \frac{\left\langle g_{\gamma_{m}}, u_{k}\right\rangle}{\left\|u_{k}\right\|} u_{k}
$$

Thus, the signal $x$ can be represented as

$$
x=\sum_{m=0}^{M-1} \frac{\left\langle u_{m}, r_{m}\right\rangle}{\left\|u_{m}\right\|} u_{m}+r_{m},
$$

where $M \ll N$ indicates that the signal can be represented by a very small amount of atoms.

2.2. The Enhanced OMP Algorithm. In essence, the OMP algorithm is also greedy iterative algorithm and requires a lot of inner product operations to obtain the best atom in each decomposition, so the computation burden is too heavy. The EOMP method introduces quantum genetic algorithm 
into the OMP algorithm. It firstly codes the parameters of dictionary of atoms in the way of quantum bit and normalizes these parameters of atoms. Secondly, it makes the inner product of the atom and the signal as the fitness function. Then, in each iteration, it makes the best solution in the process of population evolution instead of the best solution in the contemporary evolution to update quantum gate. Finally it gets the optimal value of each parameter and the best atoms. In the end, the required signal is obtained by repeating the above iterative algorithm.

Quantum Genetic Algorithm (QGA), proposed by Han and Kim [32], is a probability evolutionary algorithm combining quantum computing with genetic algorithm. Compared with the traditional genetic algorithm, it has advantages of small population size, fast convergence rate and global optimization ability, and so on. However, the population evolution is the key to this kind of evolutionary algorithm. Consequently, quantum gate has a great influence on the performance of the QGA. In this paper; the quantum rotation gate is used in population evolution operation

$$
U\left(\theta_{i}\right)=\left[\begin{array}{cc}
\cos \left(\theta_{i}\right) & -\sin \left(\theta_{i}\right) \\
\sin \left(\theta_{i}\right) & \cos \left(\theta_{i}\right)
\end{array}\right] .
$$

The process for updating qubits is summarized as follows:

$$
\left[\begin{array}{l}
\alpha_{i}^{\prime} \\
\beta_{i}^{\prime}
\end{array}\right]=U\left(\theta_{i}\right)\left[\begin{array}{l}
\alpha_{i} \\
\beta_{i}
\end{array}\right],
$$

where $\left(\alpha_{i}, \beta_{i}\right)^{T}$ and $\left(\alpha_{i}^{\prime}, \beta_{i}^{\prime}\right)^{T}$, respectively, denote the foreand-aft probability of the $i$ th quantum rotation gate updating and $\theta_{i}$ is rotation angle of the $i$ th quantum rotation gate, the size and direction of which determine the performance of the QGA.

The rotation angle of quantum gate is generally fixed and can be obtained from the table. To address this problem, a novel quantum genetic algorithm is proposed by Zhang et al. [33]. It can adaptively adjust the size of rotation angle. The rotation angle of the quantum rotation gate is described as follows:

$$
\theta_{i}=k \times f\left(\alpha_{i}, \beta_{i}\right),
$$

where $k$ has a great influence on the convergence rate of the QGA, so the value must be reasonable. In this paper, $k$ is defined as a variable related to the evolution iteration and the optimal solution, as described in (9). The EOMP can adaptively define the grid-search size in accordance with the iterations and then adaptively adjust through the comparison of the global best solution and the local best solution. Therefore, if the global best solution is better than the local best solution, it will appropriately reduce the $k$ value to diminish grid-search size and improve search precision. If the local best solution is better than the global best, it will appropriately increase the $k$ value to expand grid-search size and improve convergence rate. This guarantees the accuracy of the OMP algorithm at the same time and improves the convergence rate of the OMP algorithm

$$
k=0.5 \pi \times e^{(\bmod (i, \max (i)) / 10)}+\frac{f_{\text {best }}-f(i)}{f_{\text {best }}} \times \pi,
$$

where $\bmod (i, \max (i))$ is the remainder of $i$ and $\max (i) ; i$ and $\max (i)$ are the current iteration and the max iteration in the QGA; $f_{\text {best }}$ and $f(i)$ denote the global best solution and the local best solution, respectively.

2.3. The Dictionary Design. In addition to improving the process of the OMP algorithm, researchers have been the subjects of intense research for selection of the dictionary. Studies show that the dictionary has a great influence on the performance of the OMP algorithm $[34,35]$. To date, Gabor dictionary is widely used and has a better performance in signal processing. It is determined by four factors of $\gamma_{G}=$ $(u, m, v, \omega)$. But there exists a problem that the frequency of the time-frequency atom does not change with time. It also means that there is much truncation and mixed distortion among the signal component in the decomposition of the chirp signal. Moreover, the more parameters form, the more atom clusters and computation time are required.

A novel dictionary is recently proposed by Nagaraj et al. in the analysis of EEG signals [14] and is used to simulate the time-frequency response function of duffing oscillator through the improvement of linear frequency modulation function (Ilfm). It has a good effect on biomedical engineering due to duffing oscillator sensitive to weak characteristic signal. So, this dictionary is introduced into the proposed method for realizing the processing of mechanical fault signal.

This function of Ilfm dictionary is defined as

$$
g_{\gamma_{I L}}=e^{-0.5 \alpha^{2} t^{2}} \sin \left[2 \pi\left(f t-\beta t^{2}\right)\right]
$$

where $\alpha, f$, and $\beta$, respectively, denote time resolution, central frequency, and the rate of change of frequency. This dictionary is determined by three factors of $\gamma_{I L}=(\alpha, f, \beta)$.

2.4. The Simulation Evaluation. In this paper, the simulation platform is listed as follows: CPU (i7-4770k), Memory (8 G), and SSD $(128 \mathrm{G})$. The simulation software is MATLAB. In order to test the effectiveness and accuracy of the EOMP method, the following evaluation indicators are introduced in this paper.

(1) signal-to-error ratio (SER)

$$
\mathrm{SER}=20 \log _{10}\left(\frac{\|x\|_{2}}{\left\|r_{m}\right\|_{2}}\right)
$$

(2) comparability index $\left(C_{m}\right)$

$$
C_{m}=\frac{\left\langle x, \tilde{x}_{m}\right\rangle}{\|x\| \cdot\left\|\tilde{x}_{m}\right\|} .
$$

(3) mean square error (MSE)

$$
\mathrm{MSE}=\frac{1}{N} \sum_{m=0}^{N-1}\left(\tilde{x}_{m}-x\right)^{2}
$$




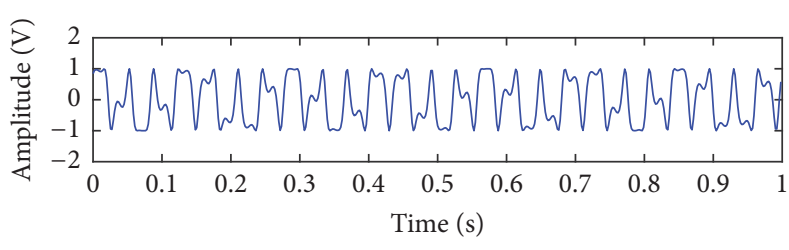

(a)

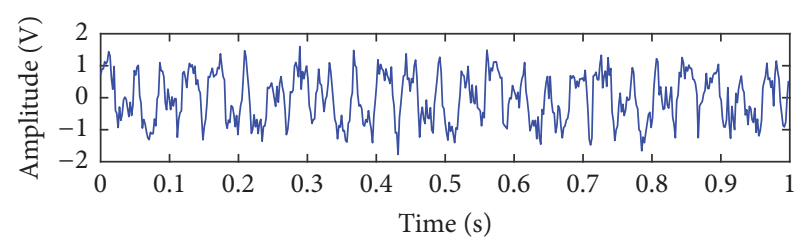

(b)

FIGURE 1: The waveform of simulated signal: (a) pure signal waveform $S_{1}(t)$ and (b) noisy signal waveform $S(t)$.

where $N$ is length of the signal, $x, r_{m}$, and $\tilde{x}_{m}$ denote the source signal, the residual after the $m$ th iteration, and the reconstruction signal after the $m$ th iteration, respectively.

The simulated signal model is described in (14) and (15) and shown in Figure 1.

$$
\begin{aligned}
S_{1}(t) & =\sin (2 \pi * 25 * t+\cos (2 \pi * 32 * t)), \\
S(t) & =S_{1}(t)+S_{2}(t),
\end{aligned}
$$

where $S_{1}(t)$ is sine frequency modulation signal, of which the frequency is $25 \mathrm{~Hz}$ and $S_{2}(t)$ is random noise, of which the amplitude is 0.3 .

2.4.1. Performance Analysis of the EOMP Method. In order to achieve a comprehensive analysis of EOMP method, Gabor dictionary and Ilfm dictionary are used to decompose and reconstruct the same simulated signal in (15). The corresponding conclusion is obtained by analysis and comparison of these results. The concrete research can be divided into the following situations:

(1) Gabor dictionary and Ilfm dictionary are used to decompose and reconstruct the simulated signal of (15) in the OMP algorithm.

(2) Gabor dictionary and Ilfm dictionary are used to decompose and reconstruct the simulated signal of (15) in the combinations of the QGA and the OMP algorithm.

(3) Gabor dictionary and Ilfm dictionary are used to decompose and reconstruct the simulated signal of (15) in the combinations of the IQGA and the OMP algorithm.

It should also be noted that the simulated noisy signal of (15) is used as the input signal, of which the sampling number and the sampling frequency are 512 and $512 \mathrm{~Hz}$. In (15), $\alpha \in$ $\left[0, \log _{2} N\right], f=[0,256](\mathrm{Hz})$, and $\beta=[-100,100](\mathrm{Hz} / \mathrm{s})$. In this paper, the population size of quantum genetic, the number of qubits, and the maximum iteration of quantum genetic are 90,10 , and 160 . The $k$ value is $0.01 \pi$ in the QGA, as shown in (9) in the IQGA.

In Figure 2(b), the simulated signal can be effectively reconstructed in six kinds of ways. However, Figures 2(a), 2(b), and 2(c) show that the method that uses Ilfm dictionary in the combinations of the IQGA and the OMP algorithm has the lowest residual, the best comparability of reconstruction, and the minimal error of reconstruction. This result indicates that this approach has fast decomposition, better reconstruction of signal, and more stability of decomposition and reconstruction. Moreover, in Figure 3, this algorithm can sharply reduce the calculation time under the same iterations. In conclusion, the EOMP method, which uses Ilfm dictionary in the combinations of the IQGA and the OMP algorithm, is optimum not only in the accuracy of reconstruction but also in reducing calculation time.

2.4.2. Analysis of Influence of Noise. The above simulation results show the EOMP method has a high degree of accuracy in reconstruction of signal. In order to further explore the performance of this algorithm, it is used to reconstruct the useful signal from the noisy signal under the condition of different strength of the noise, namely, the influence of noise. Adopting the simulated signal of (15), the EOMP method is used to decompose and reconstruct the signal under the amplitudes of the random noise such as $0.3,0.5,0.7$, and 0.9 . The corresponding conclusion is obtained by the analysis of $C_{m}$ and MSE. In this part, the simulated pure signal of (14) is used as the useful signal.

In Figures 4(a), 4(b), 4(c), and 4(d), by the comparison of the reconstructed signal and the useful signal, the optimum reconstructed signal in the EOMP algorithm is less similar to the useful signal when the noise is bigger. This result indicates the noise can truly impact on the proposed algorithm and have a growing influence on this method with bigger and bigger noise. When the noise amplitude is 0.3 , the accuracy of extraction is the best $\left(C_{m}\right.$ can be 0.9497$)$. When the noise amplitude is 0.7 , the accuracy of extraction descends $\left(C_{m}\right.$ can be 0.8494$)$. So this approach can be normally used for extraction. In the condition of the high intensity noise, we can use the preprocessing of denoising method to reduce the influence of noises. Moreover, in Figures 4(e) and 4(f), the degree of the reconstructed signal close to the useful signal decreases with increase of iterations after the degree is optimum. This result indicates that the noise may be introduced once again into the reconstructed signal with increase of iterations. Therefore the useful signal can be obtained only after few iterations, and the residual signal can be considered as noise.

\section{The Signal Feature Extraction}

In the previous section, the EOMP method can extract the useful signal from the noisy signal. Furthermore, we explore whether or not it extracts the useful signal from the 


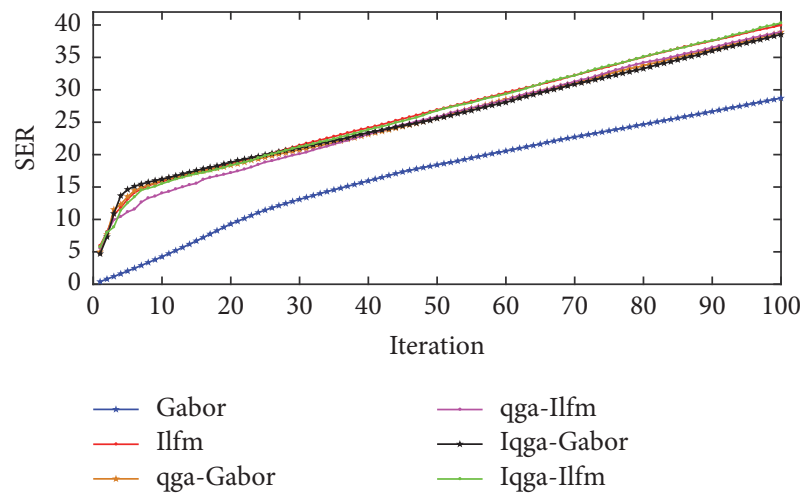

(a)

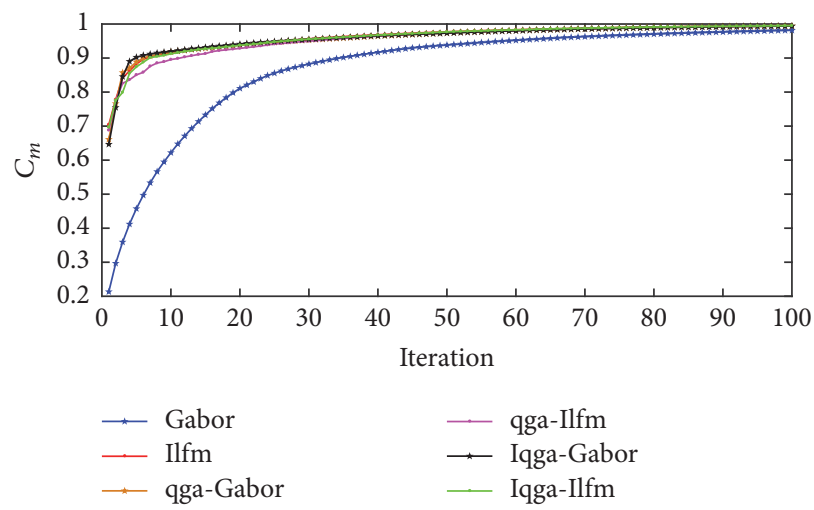

(b)

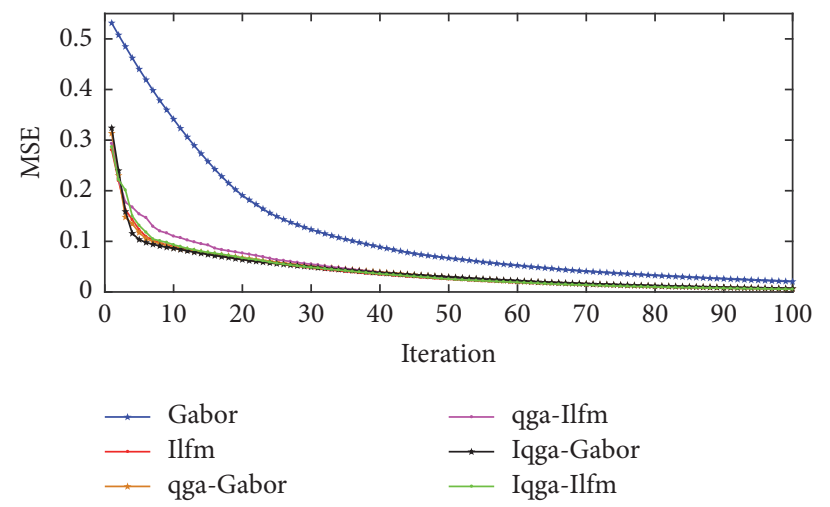

(c)

FIGURE 2: Simulated signal analysis results: Gabor dictionary and Ilfm dictionary are used to match the simulated signal 100 times in the OMP algorithm, the combinations of the QGA and the OMP algorithm, and the combinations of the IQGA and the OMP algorithm: (a) SER is changed with the iterations (the higher value shows the faster decomposition), (b) $C_{m}$ is changed with the iterations (the closer value to 1 shows the better reconstruction of signal), and (c) MSE is changed with the iterations (the lower value shows the more stability of decomposition and reconstruction).

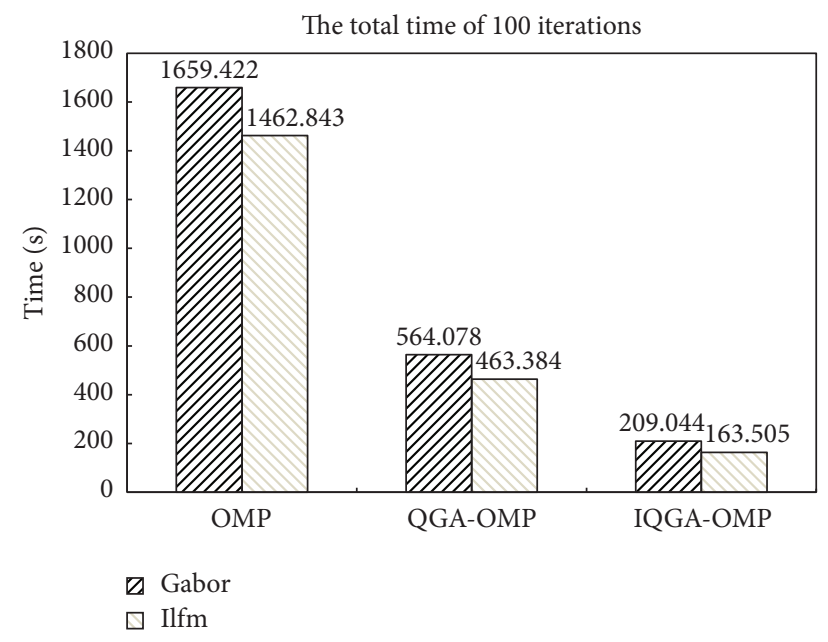

FIGURE 3

complicated signal for feature extraction in mechanical fault diagnosis.

In mechanical fault, effective extraction is critical to fault diagnose, and the impact signal has a high proportion. The accuracy of the feature extraction depends on whether or not we can find an appropriate form of basis functions (atoms). However, the actual mechanical fault signals are usually consisted by mixed sine signals and the transient impulse signal, and the effect of only using the single basis function for feature extraction is not ideal. If we can find a kind of basis functions (atoms) that can match sine component and transient impulse component, this problem can be solved.

Theoretically, the basis function (atom) of (10) can be considered as the product of the negative exponential function and the sine frequency modulation signal. So, using this basis function (atom) to decompose the mechanical vibration signal is very ideal. In order to test the effectiveness and accuracy in the feature extraction of the mixed signal, we use the simulated signal of (18) to decompose and reconstruct the signal.

The simulated signal model is described in (16), (17), and (18) and shown in Figures 5(a), 5(b), and 5(c).

$$
\begin{aligned}
& S_{1}(t)=2 * e^{\left(-0.1 * 2 \pi * 40 * t_{0}\right)} * \sin \left(2 \pi * 40 * t_{0}\right), \\
& S_{2}(t)=\sin (2 \pi * 28 * t)+1.2 * \sin (2 \pi * 41 * t),
\end{aligned}
$$


The noisy signal

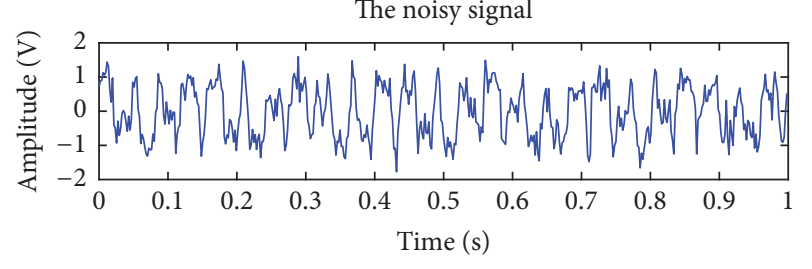

_ The noisy signal

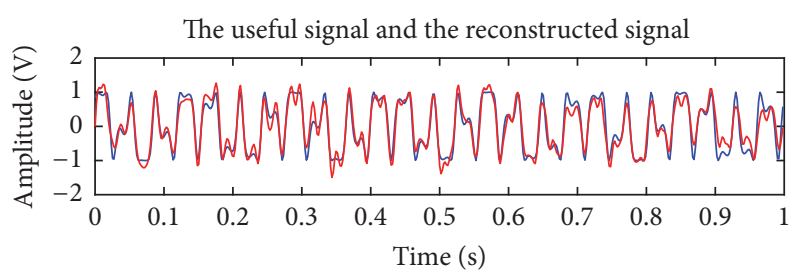

_ The useful signal

_ The reconstructed signal

(a)

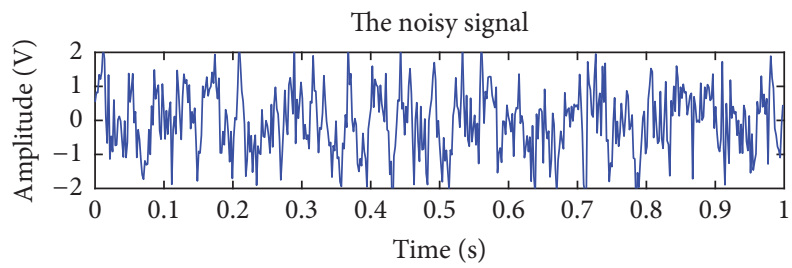

— The noisy signal

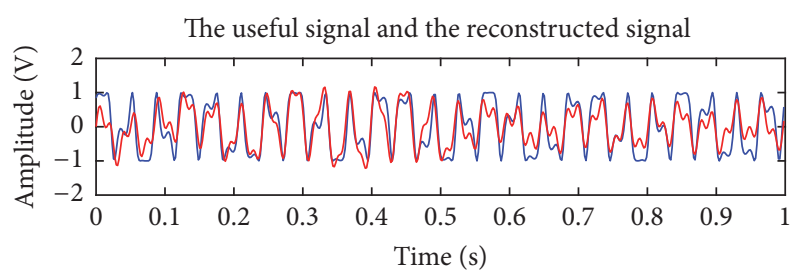

- The useful signal

_ The reconstructed signal

(c)

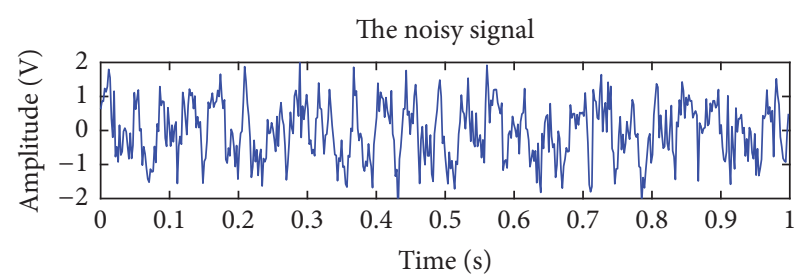

_ The noisy signal

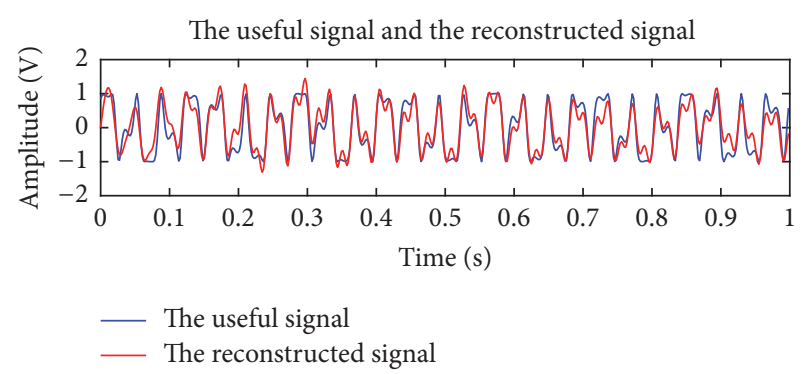

(b)

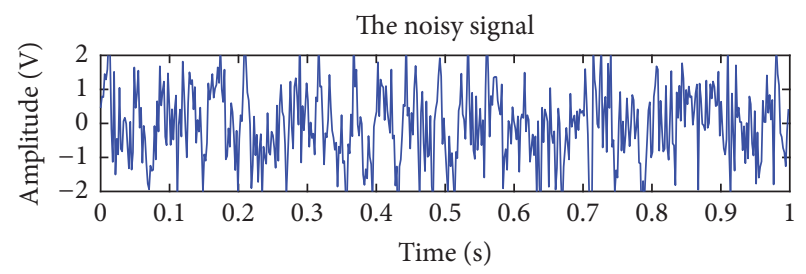

_ The noisy signal

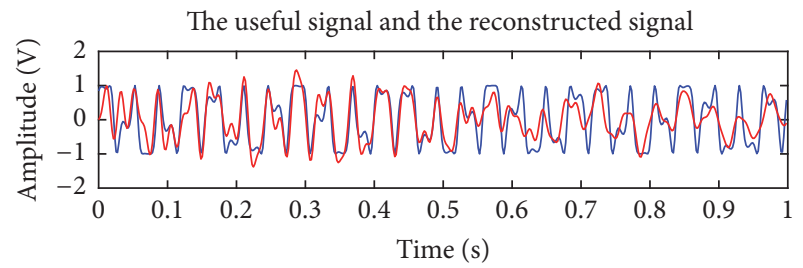

_ The useful signal

_ The reconstructed signal

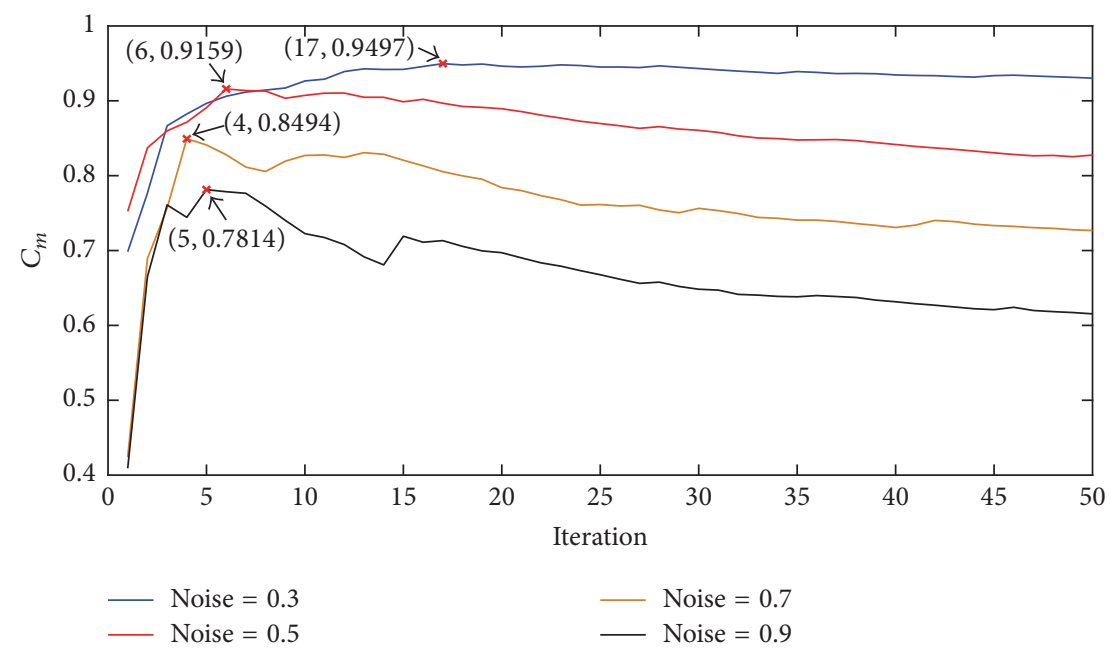

(e)

Figure 4: Continued. 


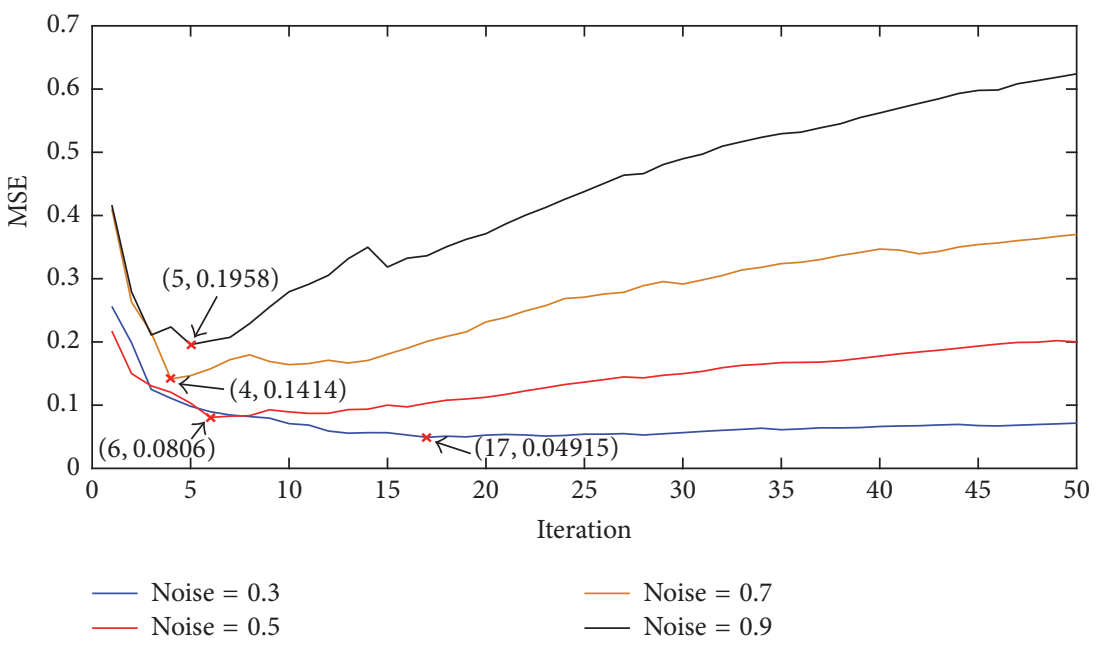

(f)

FIGURE 4: Simulated signal analysis results from the proposed method: (a) waveform of the optimum reconstructed signal under the noise amplitude of 0.3 , (b) waveform of the optimum reconstructed signal under the noise amplitude of 0.5, (c) waveform of the optimum reconstructed signal under the noise amplitude of 0.7 , (d) waveform of the optimum reconstructed signal under the noise amplitude of 0.9 , (e) $C_{m}$ is changed with the iterations, and (f) MSE is changed with the iterations.

$$
S(t)=S_{1}(t)+S_{2}(t)+S_{3}(t)
$$

where $S_{1}(t)$ is periodic impulse decay signal, of which the frequency is $40 \mathrm{~Hz}, S_{2}(t)$ is mixed sine signal, of which the frequencies are $28 \mathrm{~Hz}$ and $41 \mathrm{~Hz}$, and $S_{3}(t)$ is random noise, of which the amplitude is 0.4 .

In Figure 5(c), waveform of the periodic impulse decay signal can not be seen in time domain; at the same time, its frequency $(40 \mathrm{~Hz})$ and its corresponding sideband in frequency domain can not be found by directly obtaining FFT spectrum of the simulated signal $S(t)$. However, waveform and FFT spectrum of the extracted signal $S_{1}(t)$ in the EOMP method in Figure 5(d) are similar to the simulated signal $S_{1}(t)$ in Figure 5(a), which shows that the EOMP method can relatively accurately obtain the frequency $(40 \mathrm{~Hz})$ of the periodic impulse decay signal and its corresponding sideband in frequency domain and not completely but obviously get its waveform due to the influence of noise. Waveform and FFT spectrum of the extracted signal $S_{2}(t)$ in the EOMP method in Figure 5(e) are basically the same as that of the simulated signal $S_{2}(t)$ in Figure 5(b), which shows that the EOMP method can accurately obtain the waveform and frequency $(28 \mathrm{~Hz}$ and $40 \mathrm{~Hz}$ ) of the mixed sine signal. In conclusion, the EOMP algorithm can accurately extract the simulated fault characteristics of the periodic impulse decay signal from the mechanical fault simulated signal, which shows that the EOMP method has good effect on extraction of the impact signal.

\section{The Application in Actual Fault Diagnosis}

In order to test effectiveness of the EOMP method in the actual mechanical equipment fault diagnosis, the gear fault signal and the rolling bearing fault signal from the fault diagnosis test-bed are used to decompose and reconstruct the fault signal for obtaining the fault feature frequency components. FFT spectrum and Hilbert spectrum as traditional analysis method are used in this part.

4.1. Rolling Bearing Fault Diagnosis. The bearing fault data are obtained from the bearing fault test-bed in Case Western Reserve University. This equipment uses the deep groove ball bearing of 6205-2RS and is shown in Figure 6. The main parameters are listed as follows: the sampling number and the sampling frequency are 8192 and $12000 \mathrm{~Hz}$ and the rotating speed is $1752 \mathrm{rpm}$. And the following empirical formulas of bearing are introduced in this paper:

(1) Rotating frequency $\left(F_{r}\right)$

$$
F_{r}=\frac{r}{60} \text {. }
$$

(2) Defect frequency on inner ring $\left(F_{\text {ir }}\right)$

$$
F_{\mathrm{ir}}=F_{r} * \frac{1}{2} n\left(1+\frac{d}{D} \cos \alpha\right) .
$$

(3) Defect frequency on outer ring $\left(F_{\text {or }}\right)$

$$
F_{\text {or }}=F_{r} * \frac{1}{2} n\left(1-\frac{d}{D} \cos \alpha\right) \text {. }
$$

(4) Defect frequency on rolling element $\left(F_{\text {re }}\right)$

$$
F_{\mathrm{re}}=F_{r} * \frac{1}{2} \frac{D}{d}\left(1-\left(\frac{d}{D} \cos \alpha\right)^{2}\right) .
$$

(5) Defect frequency on cage train $\left(F_{\mathrm{ct}}\right)$

$$
F_{\mathrm{ct}}=F_{r} * \frac{1}{2}\left(1-\frac{d}{D} \cos \alpha\right)
$$



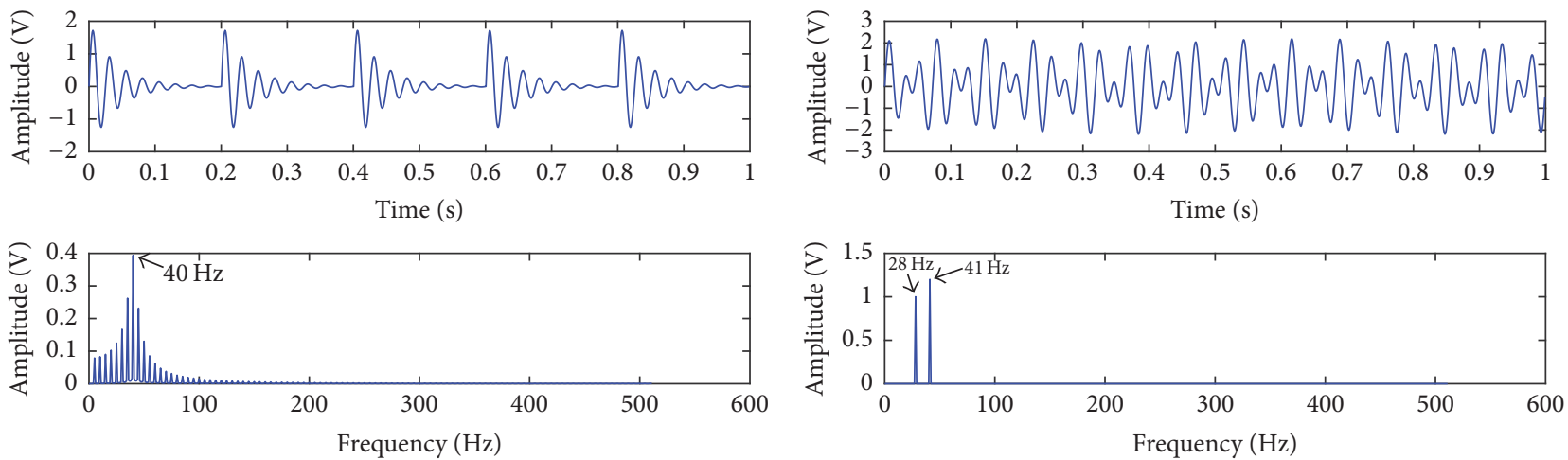

(a)

(b)
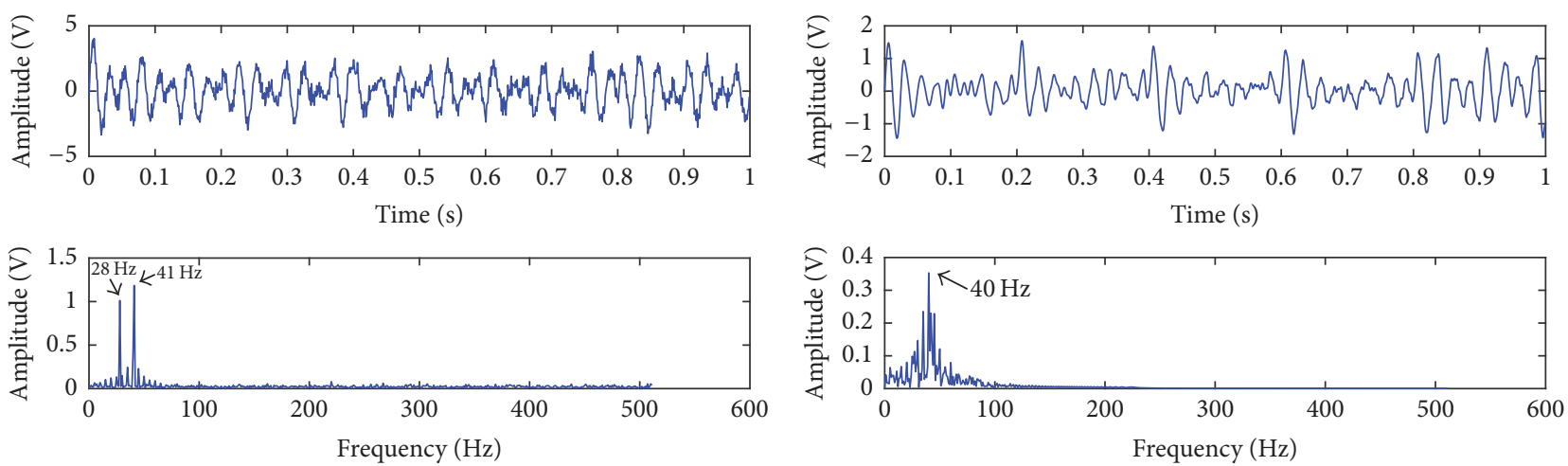

(c)

(d)
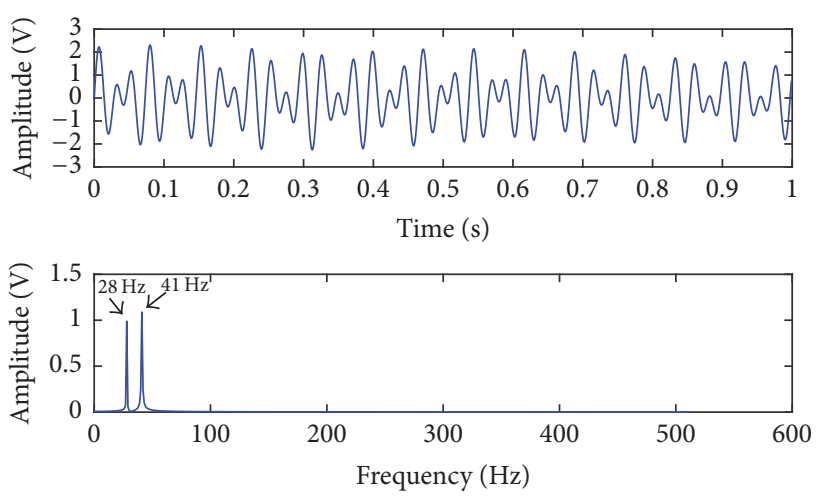

(e)

FIGURE 5: Simulated signal analysis results from the EOMP method: (a) waveform and FFT spectrum of the simulated signal $S_{1}(t)$, (b) waveform and FFT spectrum of the simulated signal $S_{2}(t)$, (c) waveform and FFT spectrum of the simulated signal $S(t)$, (d) waveform and FFT spectrum of the extracted signal $S_{1}(t)$, and (e) waveform and FFT spectrum of the extracted signal $S_{2}(t)$.

Table 1

\begin{tabular}{lcccc} 
Rotating & Inner ring & Outer ring & Rolling element & Cage train \\
\hline $29.2 \mathrm{~Hz}$ & $158.12 \mathrm{~Hz}$ & $104.68 \mathrm{~Hz}$ & $137.63 \mathrm{~Hz}$ & $11.63 \mathrm{~Hz}$
\end{tabular}

where $r$ is rotating speed of bearing and $d, D, n$, and $\alpha$ denote ball diameter, pitch diameter, number of balls, and contact angle, respectively.

According to (19), (20), (21), (22), and (23), the characteristic frequency of the bearing can be found in Table 1 .

In Figure 7(b), although the frequency of $156.7 \mathrm{~Hz}$ and its second harmonic $(314.9 \mathrm{~Hz})$ and its corresponding sideband

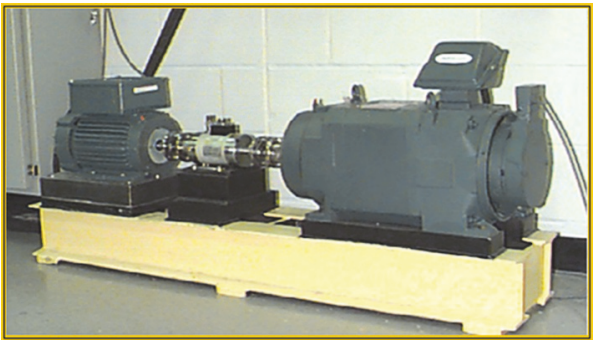

FiguRE 6

can be seen not only in FFT spectrum of the source signal but also in FFT spectrum of the reconstructed signal, the 

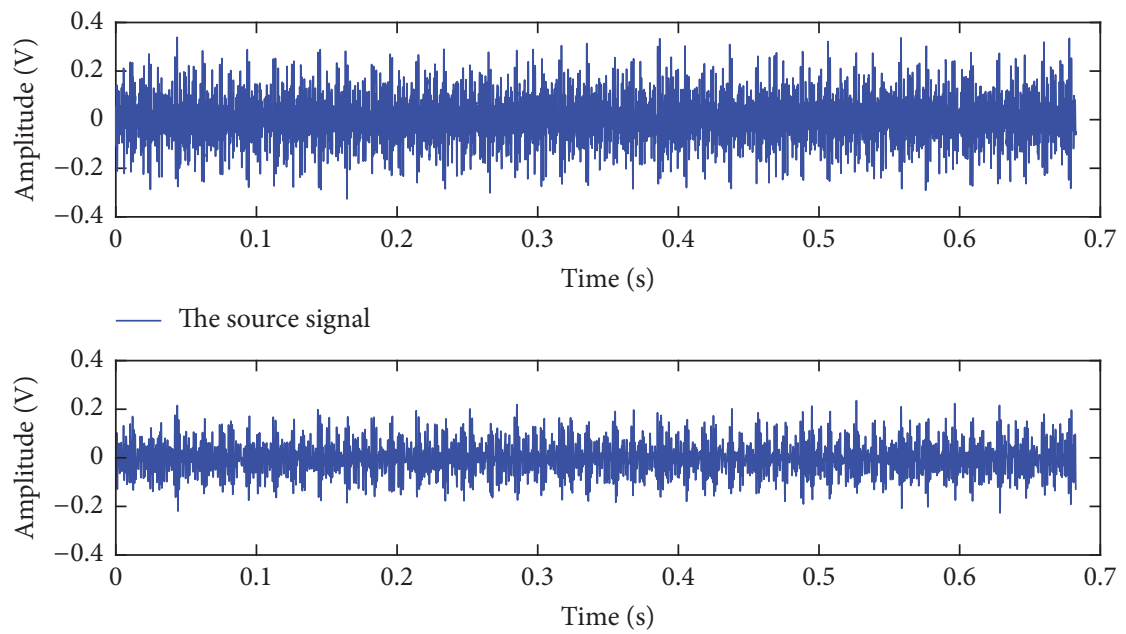

- The reconstructed signal

(a)

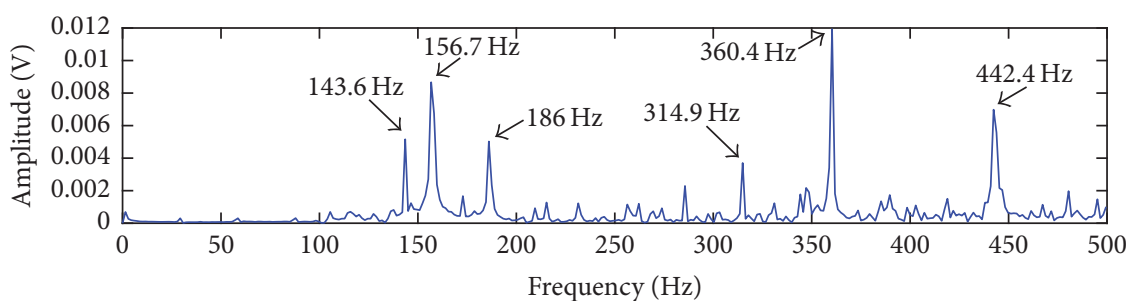

— The source signal

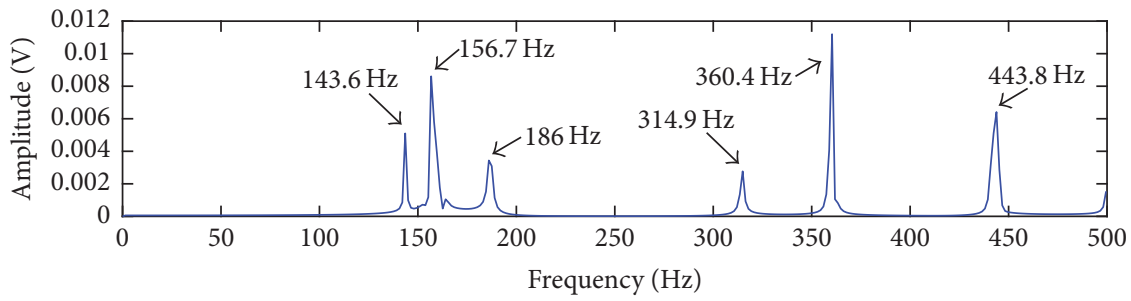

— The reconstructed signal

(b)

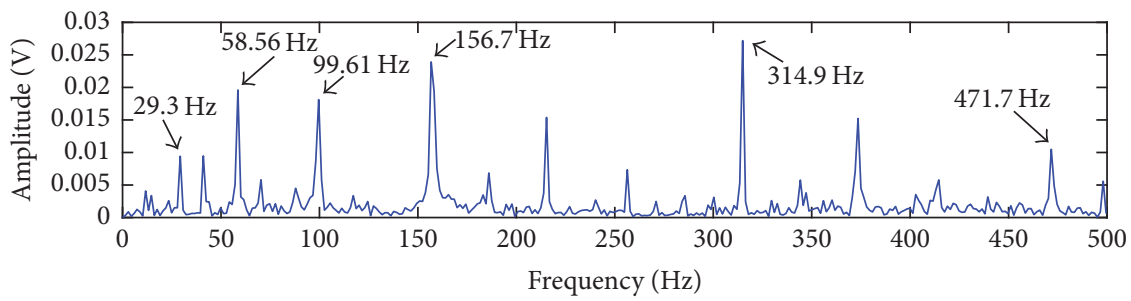

— The source signal

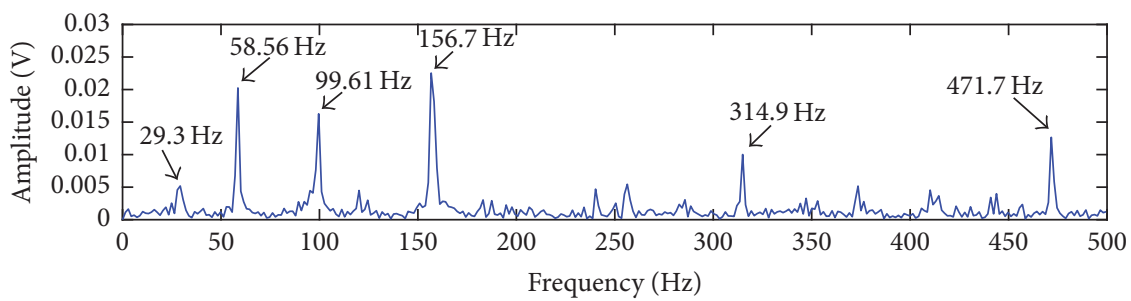

— The reconstructed signal

(c)

FIGURE 7: Bearing fault signal analysis results in the EOMP method: (a) waveform of the source signal and waveform of the reconstructed signal, (b) FFT spectrum of the source signal and FFT spectrum of the reconstructed signal, and (c) Hilbert spectrum of the source signal and Hilbert spectrum of the reconstructed signal. 

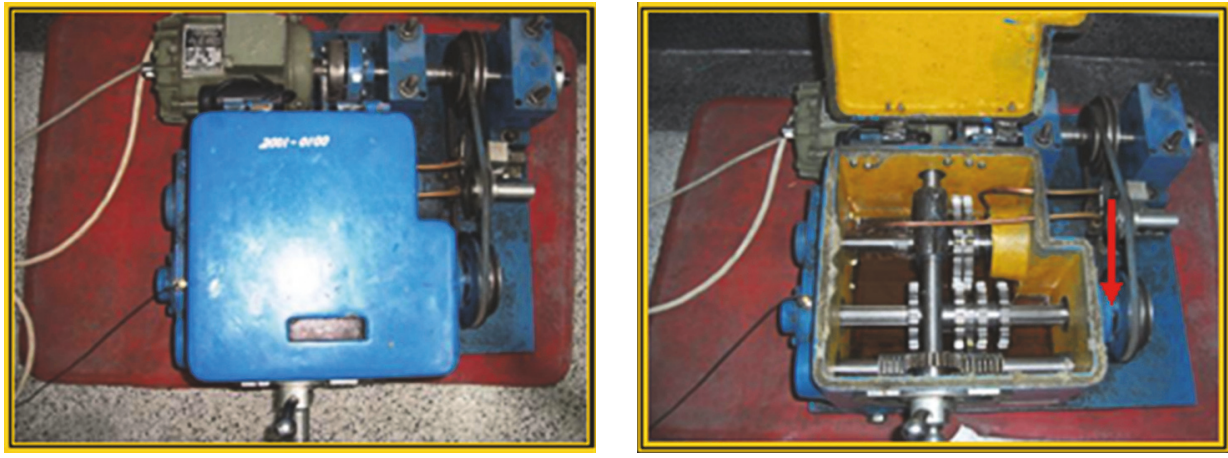

Figure 8

EOMP method can effectively diminish the influence of background noise and unrelated frequency. In Figure 7(c), the frequency of $29.3 \mathrm{~Hz}$ and its second harmonic $(58.56 \mathrm{~Hz})$, the frequency of $156.7 \mathrm{~Hz}$ and its second harmonic $(314.7 \mathrm{~Hz})$, and its third harmonic $(471.7 \mathrm{~Hz})$ and its corresponding sideband $(99.61 \mathrm{~Hz})$ can be seen not only in Hilbert spectrum of the source signal but also in Hilbert spectrum of the reconstructed signal. But the EOMP method can easily get these frequencies and effectively reduce the influence of background noise and unrelated frequency. For instance, the frequency $(156.7 \mathrm{~Hz})$ is obvious due to its highest amplitude. The frequency $29.3 \mathrm{~Hz}$ and the frequency $156.7 \mathrm{~Hz}$ are close to the rotating frequency $(29.3 \mathrm{~Hz})$ and the fault characteristic frequency of bearing inner race $(156.7 \mathrm{~Hz})$. So the fault can be preliminarily estimated as the fault of bearing inner race and this conclusion is in conformity with reality. It is concluded that the EOMP method can effectively reduce the influence of background noises and unrelated frequency compared with traditional spectrum analysis method.

4.2. Gear Fault Diagnosis. The gear fault data are obtained from the gear diagnosis fault test-bed, and this equipment is shown in Figure 8. The main parameters are listed as follows: the large gear number is 37 , the small gear number is 20 , the sampling number and the sampling frequencies are 8192 and $8192 \mathrm{~Hz}$, and the rotating speed is $855 \mathrm{rpm}$. And the following empirical formulas of gear are introduced in this paper:

(1) Rotating frequency $\left(F_{r}\right)$

$$
F_{r}=\frac{r}{60}
$$

(2) Natural frequency of gear $\left(F_{n}\right)$

$$
F_{n}=F_{r} * Z \text {, }
$$

where $r$ is rotating speed of gearing and $Z$ is tooth number of gear.

According to (24) and (25), the natural frequency of the small gear is $285 \mathrm{~Hz}$, and the rotating frequency is $14.25 \mathrm{~Hz}$.

In Figure 9(a), the EOMP method can not obviously extract the gear fault signal in time domain. However, in Figure 9(b), although the frequencies such as the natural frequency $(285 \mathrm{~Hz})$, its second harmonic $(569 \mathrm{~Hz})$ and its third harmonic $(854 \mathrm{~Hz})$, and part of these corresponding sidebands $(270 \mathrm{~Hz}, 299 \mathrm{~Hz}$, and $555 \mathrm{~Hz})$ can be seen not only in FFT spectrum of the source signal but also in FFT spectrum of the reconstructed signal, the EOMP method can easily get these useful frequencies and effectively diminish the influence of background noise and unrelated frequency. It is obviously identified that the other corresponding sidebands $(256 \mathrm{~Hz}, 541 \mathrm{~Hz}, 583 \mathrm{~Hz}, 848 \mathrm{~Hz}$, and $868 \mathrm{~Hz})$ are clearly obtained. In Figure 9(c), the frequencies such as the rotating frequency $(14 \mathrm{~Hz})$, the natural frequency $(285 \mathrm{~Hz})$, and its second harmonic $(569 \mathrm{~Hz})$ can be seen not only in Hilbert spectrum of the source signal but also in Hilbert spectrum of the reconstructed signal. But the EOMP method can easily get these frequencies and effectively reduce the influence of background noise and unrelated frequency. Obviously, these harmonic $(28 \mathrm{~Hz}$ and $85 \mathrm{~Hz})$ and these corresponding sidebands $(256 \mathrm{~Hz}, 270 \mathrm{~Hz}, 299 \mathrm{~Hz}$, and $569 \mathrm{~Hz})$ are clearly inspected. Thus, the existence of gear fault can be preliminarily estimated by frequency information. It is concluded that the EOMP method can obtain more characteristic frequency information and effectively reduce the influence of background noise and unrelated frequency.

\section{Conclusion}

An improved orthogonal matching pursuit algorithm is proposed in this paper. The main research work can be summarized as the following aspects: (1) it is novel that the EOMP method uses Ilfm dictionary in the selection of dictionary and the performance of this method is better than that of the common OMP method using Gabor dictionary in terms of accuracy and computing efficiency. (2) The proposed method combines the QGA with the OMP algorithm for high-effective atom selection, since the QGA can quickly solve the problem of multiple parameters optimization. (3) It completes the sparse representation of the original signals. Compared with the traditional OMP algorithms, the EOMP method is more sparse and efficient. By analysis of the simulated data, it is proved that the EOMP method is superior to the traditional OMP algorithms not only in the accuracy but also in computing efficiency. In addition, in the application of actual fault diagnosis, compared with the traditional spectrum analysis method, the EOMP approach 

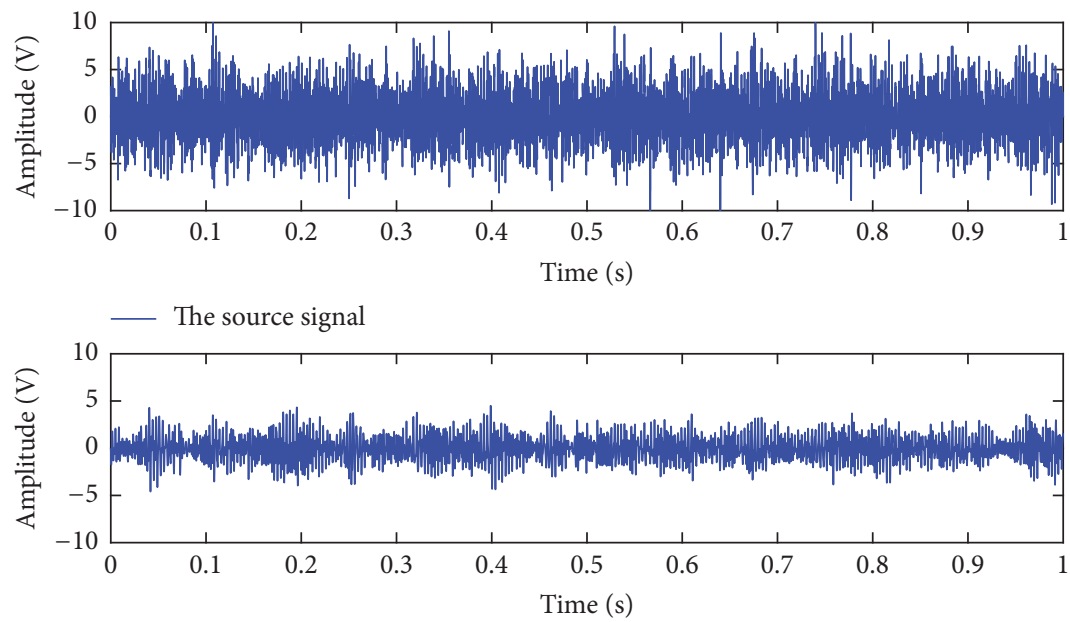

The reconstructed signal

(a)

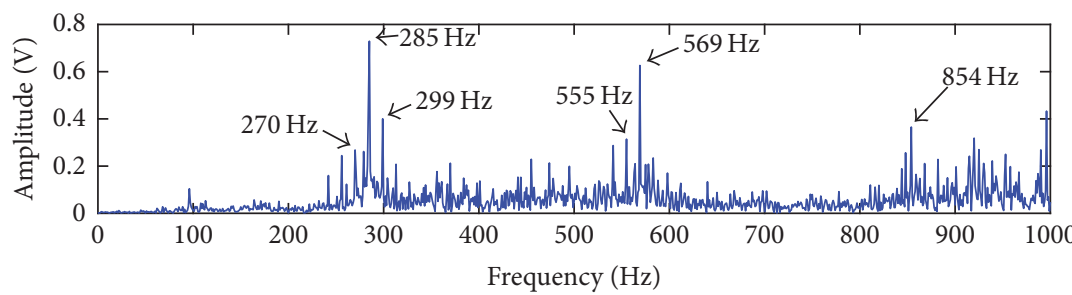

— The source signal

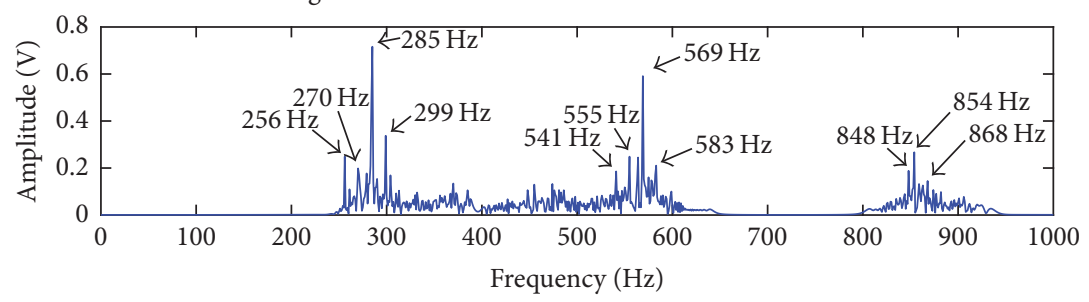

— The reconstructed signal

(b)
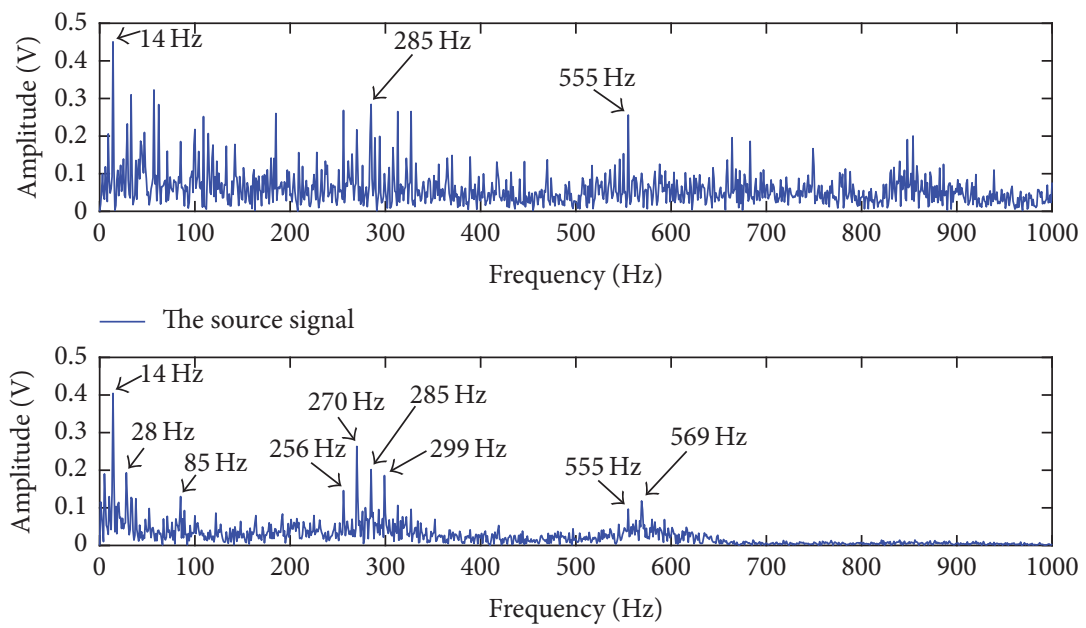

_ The reconstructed signal

(c)

FIGURE 9: Gear fault signal analysis results from the EOMP method: (a) waveform of the source signal and waveform of the reconstructed signal, (b) FFT spectrum of the source signal and FFT spectrum of the reconstructed signal, and (c) Hilbert spectrum of the source signal and Hilbert spectrum of the reconstructed signal. 
can easily obtain the fault characteristic information and effectively reduce the interference of background noises and unrelated frequency. These results demonstrated that the EOMP algorithm is feasible and effective in actual mechanical equipment fault diagnosis.

\section{Conflicts of Interest}

The authors declare that there are no conflicts of interest regarding the publication of this paper.

\section{Acknowledgments}

This work was supported by the National Natural Science Foundation of China (no. 51475339) and the Natural Science Foundation of Hubei province (no. 2016CFA042).

\section{References}

[1] L. Zhang, J. Xu, J. Yang, D. Yang, and D. Wang, "Multiscale morphology analysis and its application to fault diagnosis," Mechanical Systems and Signal Processing, vol. 22, no. 3, pp. 597610, 2008.

[2] D. Gabor, "Theory of communication. Part 1: the analysis of information," Journal of the Institution of Electrical Engineers-Part III: Radio and Communication Engineering, vol. 93, no. 26, pp. 429-441, 1946.

[3] S. Mallat, A Wavelet Tour of Signal Processing, Academic Press, San Diego, Calif, USA, 2nd edition, 1998.

[4] N. E. Huang, Z. Shen, S. R. Long et al., "The empirical mode decomposition and the Hilbert spectrum for nonlinear and non-stationary time series analysis," The Royal Society of London A, vol. 454, pp. 903-995, 1998.

[5] F. Riaz, A. Hassan, S. Rehman, I. K. Niazi, and K. Dremstrup, "EMD-based temporal and spectral features for the classification of EEG signals using supervised learning," IEEE Transactions on Neural Systems and Rehabilitation Engineering, vol. 24 , no. 1, pp. 28-35, 2016.

[6] J. Cai and X. Li, "Gear fault diagnosis based on empirical mode decomposition and 1.5 dimension spectrum," Shock and Vibration, vol. 2016, Article ID 5915762, 10 pages, 2016.

[7] Y. Chen, C. Zhou, J. Yuan, and Z. Jin, "Applications of empirical mode decomposition in random noise attenuation of seismic data," Journal of Seismic Exploration, vol. 23, no. 5, pp. 481-495, 2014.

[8] L. Zhao, W. Yu, and R. Yan, "Gearbox fault diagnosis using complementary ensemble empirical mode decomposition and permutation entropy," Shock and Vibration, vol. 2016, Article ID 3891429, 8 pages, 2016.

[9] A. A. Tabrizi, L. Garibaldi, A. Fasana, and S. Marchesiello, "Performance improvement of ensemble empirical mode decomposition for roller bearings damage detection," Shock and Vibration, vol. 2015, Article ID 964805, 10 pages, 2015.

[10] F. J. Wu and L. S. Qu, "An improved method for restraining the end effect in empirical mode decomposition and its applications to the fault diagnosis of large rotating machinery," Journal of Sound and Vibration, vol. 314, no. 3-5, pp. 586-602, 2008.

[11] S. G. Mallat and Z. Zhang, "Matching pursuits with timefrequency dictionaries," IEEE Transactions on Signal Processing, vol. 41, no. 12, pp. 3397-3415, 1993.
[12] H. Wang, R. Zhao, and Y. Cen, "Rank adaptive atomic decomposition for low-rank matrix completion and its application on image recovery," Neurocomputing, vol. 145, pp. 374-380, 2014.

[13] H. Yin, "Sparse representation with learned multiscale dictionary for image fusion," Neurocomputing, vol. 148, pp. 600-610, 2015.

[14] S. B. Nagaraj, N. Stevenson, W. Marnane, G. Boylan, and G. Lightbody, "A novel dictionary for neonatal EEG seizure detection using atomic decomposition," in Proceedings of the 2012 Annual International Conference of the IEEE Engineering in Medicine and Biology Society (EMBC), pp. 1073-1076, San Diego, Calif, USA, September 2012.

[15] S. B. Nagaraj, N. J. Stevenson, W. P. Marnane, G. B. Boylan, and G. Lightbody, "Neonatal seizure detection using atomic decomposition with a novel dictionary," IEEE Transactions on Biomedical Engineering, vol. 61, no. 11, pp. 2724-2732, 2014.

[16] S. Wang, Y. Chen, and Y. Bai, "A surveillance video compression algorithm based on regional dictionary," in Proceedings of the 2016 8th International Conference on Computer and Automation Engineering, ICCAE 2016, aus, March 2016.

[17] Y. Xiong, D. Guan, C. Li, and Z. Jiang, "Application of fast matching pursuit decomposition technology based on anisotropic structure-oriented filtering method to complex fault block of Bohai Bay Basin," in Proceedings of the SEG Technical Program Expanded Abstracts 2016, pp. 4725-4729, Dallas, Tex, USA.

[18] X. Feng, X. Zhang, C. Liu, and Q. Lu, "Single-channel and multi-channel orthogonal matching pursuit for seismic trace decomposition," Journal of Geophysics and Engineering, vol. 14, no. 1, pp. 90-99, 2017.

[19] S. S. Chen, D. L. Donoho, and M. A. Saunders, "Atomic decomposition by basis pursuit," SIAM Journal on Scientific Computing, vol. 20, no. 1, pp. 33-61, 1998.

[20] P. S. Huggins and S. W. Zucker, "Greedy basis pursuit," IEEE Transactions on Signal Processing, vol. 55, no. 7, part 2, pp. 37603772, 2007.

[21] D. Needell and R. Vershynin, "Uniform uncertainty principle and signal recovery via regularized orthogonal matching pursuit," Foundations of Computational Mathematics. The Journal of the Society for the Foundations of Computational Mathematics, vol. 9, no. 3, pp. 317-334, 2009.

[22] D. Needell and R. Vershynin, "Greedy signal recovery and uncertainty principles," in Proceedings of the Computational Imaging VI, usa, January 2008.

[23] D. L. Donoho, Y. Tsaig, I. Drori, and J.-L. Starck, "Sparse solution of underdetermined systems of linear equations by stagewise orthogonal matching pursuit," Institute of Electrical and Electronics Engineers. Transactions on Information Theory, vol. 58, no. 2, pp. 1094-1121, 2012.

[24] D. Needell and J. A. Tropp, "Iterative signal recovery from incomplete and inaccurate samples," Applied and Computational Harmonic Analysis, vol. 26, no. 3, pp. 301-321, 2009.

[25] E. J. Candes, P. R. Charlton, and H. Helgason, "Detecting highly oscillatory signals by chirplet path pursuit," Applied and Computational Harmonic Analysis, vol. 24, no. 1, pp. 14-40, 2008.

[26] F. Peng, D. Yu, and J. Luo, "Sparse signal decomposition method based on multi-scale chirplet and its application to the fault diagnosis of gearboxes," Mechanical Systems and Signal Processing, vol. 25, no. 2, pp. 549-557, 2011.

[27] C. Xu, C. Wang, and J. Gao, "Instantaneous frequency identification using adaptive linear chirplet transform and matching 
pursuit," Shock and Vibration, vol. 2016, Article ID 1762010, 2016.

[28] J. Luo, S. Zhang, M. Zhong, and Z. Lin, "Order spectrum analysis for bearing fault detection via joint application of synchrosqueezing transform and multiscale chirplet path pursuit," Shock and Vibration, vol. 2016, Article ID 2976389, 2016.

[29] H. Wang, J. Chen, and G. Dong, "Fault diagnosis method for rolling bearing's weak fault based on minimum entropy deconvolution and sparse decomposition," Jixie Gongcheng Xuebao/Journal of Mechanical Engineering, vol. 49, no. 1, pp. 8894, 2013.

[30] B. Yan and F. Zhou, "Initial fault identification of bearing based on coherent cumulant stagewise orthogonal matching pursuit," Jixie Gongcheng Xuebao/Journal of Mechanical Engineering, vol. 50, no. 13, pp. 88-96, 2014.

[31] Y. C. Pati, R. Rezaiifar, and P. S. Krishnaprasad, "Orthogonal matching pursuit: recursive function approximation with applications to wavelet decomposition," in Proceedings of the 27th Asilomar Conference on Signals, Systems and Computers, vol. 1, pp. 40-44, Pacific Grove, Calif, USA, November 1993.

[32] K. H. Han and J. H. Kim, "Quantum-inspired evolutionary algorithm for a class of combinatorial optimization," IEEE Transactions on Evolutionary Computation, vol. 6, no. 6, pp. 580-593, 2002.

[33] G. Zhang, N. Li, W. Jin, and L. Hu, "A novel, quantum genetic algorithm and its application," Acta Electronica Sinica, vol. 32, no. 3, pp. 476-479, 2004.

[34] P. D. Swami and A. Jain, "Image denoising by supervised adaptive fusion of decomposed images restored using wave atom, curvelet and wavelet transform," Signal, Image and Video Processing, vol. 8, no. 3, pp. 443-459, 2014.

[35] Y. Wang, B. Zhao, and Y. Jiang, "Inverse synthetic aperture radar imaging of targets with complex motion based on cubic Chirplet decomposition," IET Signal Processing, vol. 9, no. 5, pp. 419-429, 2015. 


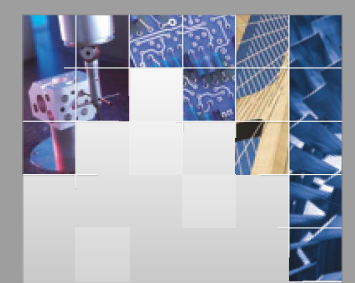

\section{Enfincering}
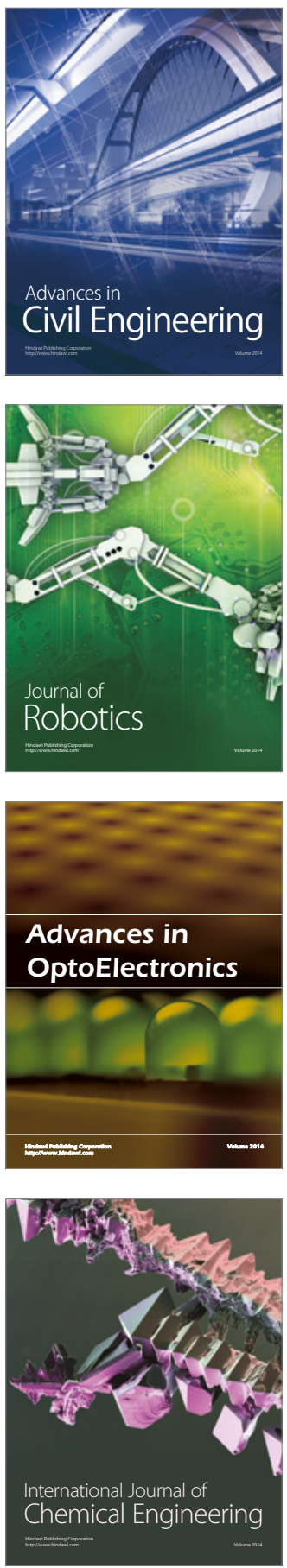

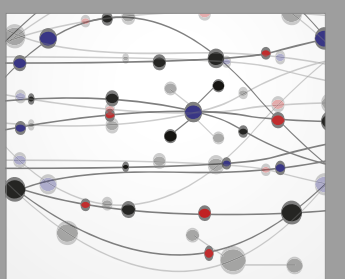

The Scientific World Journal

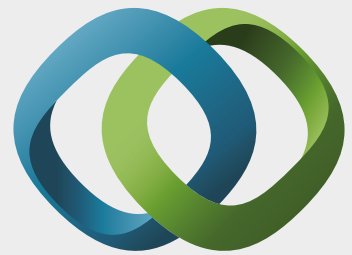

\section{Hindawi}

Submit your manuscripts at

https://www.hindawi.com
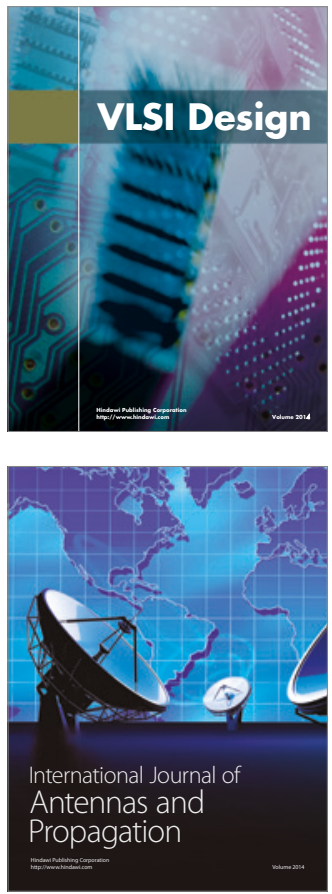

\section{Rotating}

Machinery
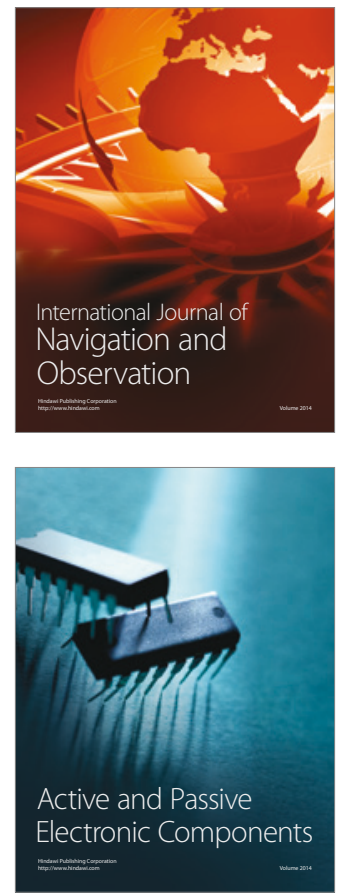
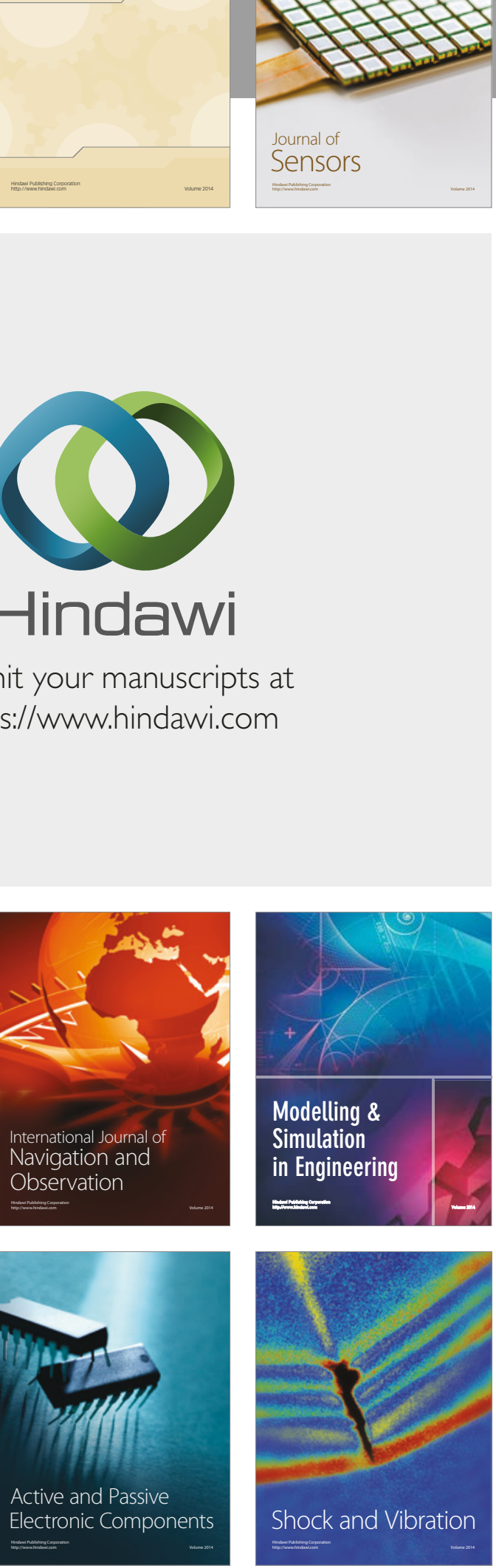
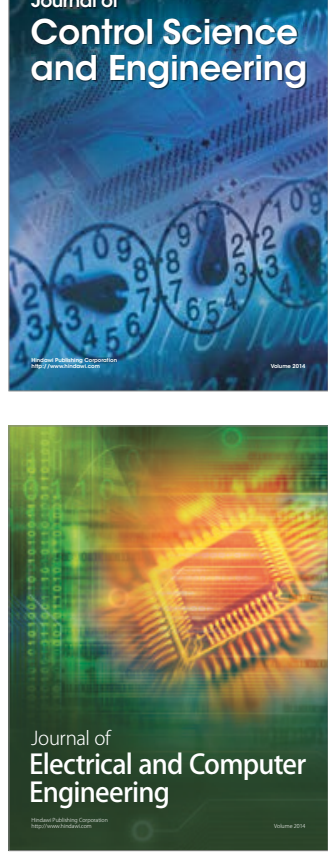

Distributed

Journal of

Control Science

and Engineering
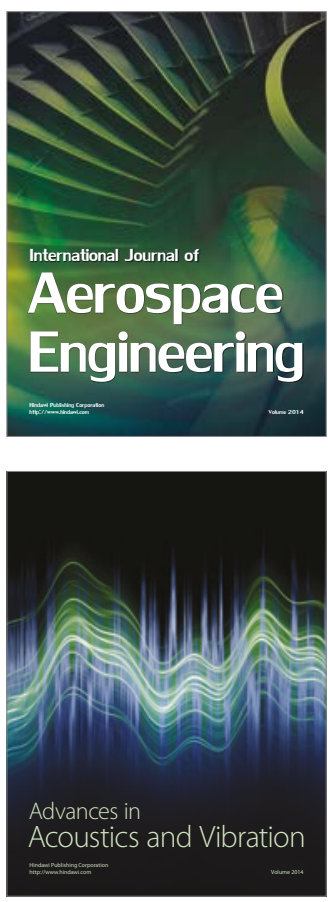

Sensor Networks 